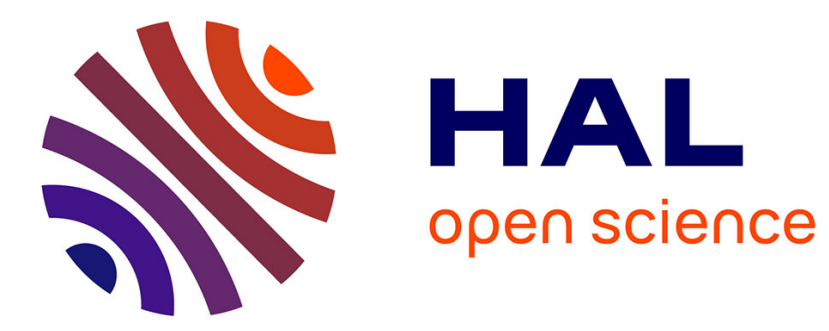

\title{
On the Joint Use of Time Reversal and POPS-OFDM for $5 \mathrm{G}$ Systems
}

Wafa Khrouf, Zeineb Hraiech, Fatma Abdelkefi, Mohamed Siala, Matthieu

Crussière

\section{- To cite this version:}

Wafa Khrouf, Zeineb Hraiech, Fatma Abdelkefi, Mohamed Siala, Matthieu Crussière. On the Joint Use of Time Reversal and POPS-OFDM for 5G Systems. VTC Fall IEEE 86th Vehicular Technology Conference, Sep 2017, Toronto, Canada. hal-01763320

\section{HAL Id: hal-01763320 \\ https://hal.science/hal-01763320}

Submitted on 10 Apr 2018

HAL is a multi-disciplinary open access archive for the deposit and dissemination of scientific research documents, whether they are published or not. The documents may come from teaching and research institutions in France or abroad, or from public or private research centers.
L'archive ouverte pluridisciplinaire HAL, est destinée au dépôt et à la diffusion de documents scientifiques de niveau recherche, publiés ou non, émanant des établissements d'enseignement et de recherche français ou étrangers, des laboratoires publics ou privés. 


\title{
On the Joint Use of Time Reversal and POPS-OFDM for 5G Systems
}

\author{
Wafa Khrouf, Zeineb Hraiech, Fatma Abdelkefi, Mohamed Siala and Matthieu Crussière* \\ MEDIATRON Laboratory, Higher School of Communications of Tunis (SUP'COM), 2083 Ariana, Tunisia \\ Emails: \{wafa.khrouf, zeineb.hraiech, fatma.abdelkefi, mohamed.siala\}@supcom.tn \\ *INSA of Rennes, IETR, UMR 6164, France \\ Email: matthieu.crussiere@insa-rennes.fr
}

\begin{abstract}
This paper investigates the efficiency of the combination of the Ping-pong Optimized Pulse Shaping-Orthogonal Frequency Division Multiplexing (POPS-OFDM) algorithm with the Time Reversal (TR) technique. This algorithm optimizes the transmit and receive OFDM waveforms with a significant reduction in the system Inter-Carrier Interference (ICI)/InterSymbol Interference (ISI) and guarantees maximal Signal to Interference plus Noise Ratio (SINR) for realistic mobile radio channels in 5G Systems. To this end, we characterize the scattering function of the TR channel and we derive the closedform expression of the SINR as a Generalized Rayleigh Quotient. Numerical analysis reveals a significant gain in SINR and OutOf-Band (OOB) emissions, brought by the proposed TR-POPSOFDM approach.
\end{abstract}

Index Terms-Waveform Optimization, Waveform Design, Pulse Shaping, POPS-OFDM, Signal to Interference plus Noise Ratio (SINR), Time Reversal

\section{INTRODUCTION}

OFDM modulation has witnessed a considerable interest from both academia and industry. This is due to many advantages such as low complexity receiver, simplicity and efficient equalization structure. Indeed, it has been adopted in various wired and wireless standards, such as ADSL, DVBT, Wimax, WiFi, and LTE. Nevertheless, in its present form, OFDM presents several shortcomings and as such it is not capable of guaranteeing the quality of service of new and innovative applications and services that will be brought by $5 \mathrm{G}$ systems. In fact, it has a high spectral leakage and it requires strict frequency synchronization because it uses a rectangular waveform in time, leading to significant sidelobes in frequency [2]. As a consequence, any lack of perfect frequency synchronization, to be expected from most of the innovative Machine Type Communications (MTC) in 5G, causes important InterCarrier Interferences (ICI). In addition to that, a variety of services and new applications will be provided by $5 \mathrm{G}$ systems, such as high data-rate wireless connectivity, which requires large spectral and energy efficiency, and Internet of Things (IoT), requiring robustness to time synchronization errors [3].

In order to overcome OFDM limitations and meet 5G requirements, various modulations have been suggested in the literature such as Generalized Frequency Division Multiplexing (GFDM), Universal Filtered Multi-Carrier (UFMC) and Filter Bank Multi-Carrier (FBMC), which are proposed in 5GNOW project [5]. It is shown at [5] that GFDM offers high flexibility for access to fragmented spectrum and low OutOf-Band (OOB) emissions. However, in contrast to UFMC, GFDM has a low robustness to frequency synchronization errors in the presence of Doppler spread. Moreover, like UFMC [4], FBMC has a high spectral efficiency and a good robustness to ICI. Nevertheless, FBMC, because of its long shaping filters, cannot be used in the case of low latency, sporadic traffic and small data packets transmission. Furthermore, authors in [1] and [6] propose new class of waveforms, namely POPSOFDM, which iteratively maximize the SINR in order to create optimal waveforms at the transmitter $(\mathrm{Tx})$ and the receiver $(\mathrm{Rx})$ sides. The obtained waveforms are well localized in time and frequency domains and they are able to reduce the ISI and the ICI as they are not sensitive to time and frequency synchronization errors. Another alternative which aims to reduce interference especially in time, caused by highly dispersive channels, is the time reversal (TR) technique which has been recently proposed for wireless communications systems. Its time and space focusing properties make it an attractive candidate for green and multiuser communications [7]. In fact, it reduces ISI at the Rx side and it mitigates the channel delay spread.

This paper aims to design new waveforms for $5 \mathrm{G}$ systems by combining the benefits of POPS and TR techniques in terms of interference resilience. For this end, we analyze the corresponding system and we derive the SINR expression. We also evaluate the performances of the proposed approach in terms of SINR and OOB emissions.

The remaining of this paper is organized as follows. In Section III, we present the system model. In Section IV, we focus on the derivation of the SINR expression for TR systems and describe the TR-POPS-OFDM algorithm for waveforms design. Section $\mathrm{V}$ is dedicated to the illustration of the obtained optimization results and shed light on the efficiency of the proposed TR-POPS-OFDM approach. Finally, Section VI presents conclusion and perspectives to our work.

\section{NOTATIONS}

Boldface lower and upper case letters refer to vectors and matrices, respectively. The superscripts . ${ }^{*}$ and.$^{T}$ denote the element-wise conjugation and the transpose of a vector or matrix, respectively. We denote by $\mathbf{v}=$ $\left(\ldots, v_{-2}, v_{-1}, v_{0}, v_{1}, v_{2}, \ldots\right)^{T}=\left(v_{q}\right)_{q \in \mathbb{Z}}=\left(v_{q}\right)_{q}$ the infinite 
vector $\mathbf{v}$. The last notation, $\left(v_{q}\right)_{q}$, with the set of values taken by $q$ is not explicitly specified, that means that $q$ spans $\mathbb{Z}$. Let $\mathbf{M}=\left(M_{p q}\right)_{p \in \mathbb{Z}, q \in \mathbb{Z}}=\left(M_{p q}\right)_{p q}$ refers to the infinite matrix M. The matrix shift operator $\Sigma_{k}(\cdot)$ shifts all matrix entries by $k$ parallel to the main diagonal of the matrix, i.e. if $\mathbf{M}=\left(M_{p q}\right)_{p q}$ is a matrix with $(p, q)$-th entry $M_{p q}$, then $\boldsymbol{\Sigma}_{k}(\mathbf{M})=\left(M_{p-k, q-k}\right)_{p q}$. The symbol $\otimes$ is the convolution operator of two vectors and the symbol $\odot$ is the componentwise product of two vectors or matrices. We denote by $\mathbb{E}$ the expectation operator and by $|$.$| the absolute value.$

\section{SYSTEM MODEL}

In this Section, we first present the TR principal. Then, we describe the channel and system models in which we will apply our approach.

\section{A. Time Reversal Principle}

The Time Reversal (TR) principle [8], [9], comes from the acoustic research field and allows a wave to be localized in time and space. Such a technique can be exploited to separate users, addressed simultaneously on the same frequency band, by their different positions in space.

The use of TR in transmission systems has generated a particular excitement as it allows, from a very high temporal dispersion channel, to obtain an ideal pulse in time and in space. This property has several useful advantages in wireless communications, among which we cite the followings:

- Negligible or null ISI brought a nearly "no memory" equivalent channel.

- Minimum inter-user interference thanks to space power localization, with received negligible power outside a focal spot targeted to a given Rx.

- Physical-layer-secured data transmission towards a desired user, as other users located outside of the focal spot of the targeted user will receive only a few power.

TR integration into a telecommunication system is very simple. It consists in applying a filter on the transmitted signal. We suppose that we have a perfect knowledge of the transmission channel and that it is invariant between the instants of its measurements and the application of the TR at the Tx side. This filter is made up of the Channel Impulse Response (CIR) reversed in time and conjugated. It has the form of a matched filter to the propagation channel which guarantees optimal reception in terms of Signal to Noise Ratio (SNR). Then, the transmitted signal will cross an equivalent filter equal to the convolution between the channel and its time reversed version.

\section{B. Channel Model}

We consider a Wide Sense Stationary Uncorrelated Scattering (WSSUS) channel in order to have more insights on the TR-POPS-OFDM performances in the general case. To simplify the derivations, we consider a discrete time system. We denote by $T_{s}$ the sampling period and by $R_{s}=\frac{1}{T_{s}}$ the sampling rate. We suppose that the channel is composed of $K$ paths and that the Tx has a perfect knowledge of the channel state at any time. Note that this hypothesis is realistic in the case of low Doppler spread. Let $\mathbf{h}^{(p)}=$ $\left(h_{0}^{(p)}, h_{1}^{(p)}, \ldots, h_{K-1}^{(p)}\right)^{T}$ be the channel discrete version at instant $p$ such as $h_{l}^{(p)}=\sum_{m=0}^{M-1} h_{l m} e^{j 2 \pi \nu_{l m} p T_{s}}$ is the path corresponding to a delay $l T_{s}$, where $M$ is the number of Doppler rays, $h_{l m}$ and $\nu_{l m}$ denote respectively the amplitude and the Doppler frequency of the $l^{\text {th }}$ path and the $m^{\text {th }}$ Doppler ray. The ray amplitudes, $h_{l m}$, are supposed to be centered, independent and identically distributed complex Gaussian variables with average powers $\pi_{l m}=\mathbb{E}\left[\left|h_{l m}\right|^{2}\right]$. We denote by $\pi_{l}=\sum_{m=0}^{M-1} \pi_{l m}$, where $\sum_{l=0}^{K-1} \pi_{l}=1$.

The channel time reversed version at the instant $p$ can be written as:

$$
\mathbf{g}^{(p)}=\left(h_{K-1}^{(p) *}, \ldots, h_{1}^{(p) *}, h_{0}^{(p) *}\right)^{T}
$$

When we apply the TR technique at the Tx in a Single Input Single Output (SISO) system, the equivalent channel, experienced by the transmission at time instant $p T_{s}$ at the $\mathrm{Rx}$, could be seen as the convolution between the channel and its time reversed version, as follows:

$$
\begin{aligned}
\mathbf{H}^{(p)} & =\mathbf{h}^{(p)} \otimes \mathbf{g}^{(p)} \\
& =\left(H_{-(K-1)}^{(p)}, \ldots, H_{-1}^{(p)}, H_{0}^{(p)}, H_{1}^{(p)}, \ldots, H_{K-1}^{(p)}\right)^{T},
\end{aligned}
$$

where $H_{k}^{(p)}=\sum_{l=0}^{K-1-|k|} \sum_{m, m^{\prime}=0}^{M-1} f\left(k, l, m, m^{\prime}\right)$, such as:

$f\left(k, l, m, m^{\prime}\right)= \begin{cases}h_{l m}^{*} h_{l+k, m^{\prime}} e^{j 2 \pi\left(\nu_{l+k, m^{\prime}}-\nu_{l m}\right) p T_{s}}, & \text { if } k \geq 0 \\ h_{l m} h_{l-k, m^{\prime}}^{*} e^{-j 2 \pi\left(\nu_{l-k, m^{\prime}}-\nu_{l m}\right) p T_{s}}, \text { else. }\end{cases}$

It should be noted that $H_{-k}^{(p)}=H_{k}^{(p) *}$, which means that the channel is Hermitian symmetric, and that the equivalent aggregate channel coefficients, $H_{k}^{(p)}$, are still decorrelated, as in the actual channel.

\section{OFDM System}

In this paper, we consider a discrete time version of the waveforms to simplify the theoretical derivations that will be investigated.

Let $T$ and $F$ refer to the OFDM symbol duration and the frequency separation between two adjacent subcarriers respectively. The sampling period is equal to $T_{s}=\frac{T}{N}$ where $N \in \mathbb{N}$. We denote by $\delta=\frac{1}{F T}=\frac{Q}{N}$ the time-frequency lattice density, where $Q=\frac{1}{T_{s} F} \leq N$ is the number of subcarriers.

We denote by $\mathbf{e}=\left(e_{q}\right)_{q}$ the sampled version of the transmitted signal at time $q T_{s}$, with a sampling rate $R_{s}=\frac{1}{T_{s}}$, expressed as:

$$
\mathbf{e}=\sum_{m, n} a_{m n} \boldsymbol{\varphi}_{m n}
$$

where $\varphi_{m n}=\left(\varphi_{q-n N}\right)_{q} \odot\left(e^{j 2 \pi m q / Q}\right)_{q}$ is the time and frequency shifted version of the OFDM transmit prototype waveform, $\boldsymbol{\varphi}=\left(\varphi_{q}\right)_{q}$, used to transmit the symbol $a_{m n}$. We suppose that the transmitted symbols are decorrelated, with zero mean and energy equal to $E=\mathbb{E}\left[\left|a_{m n}\right|^{2}\right]\|\varphi\|^{2}$. 
The received signal is expressed as:

$$
\mathbf{r}=\sum_{m n} a_{m n} \tilde{\boldsymbol{\varphi}}_{m n}+\mathbf{n},
$$

where $\left[\tilde{\boldsymbol{\varphi}}_{m n}\right]_{q}=\sum_{k=-(K-1)}^{K-1} H_{k}^{(q)}\left[\boldsymbol{\varphi}_{m n}\right]_{q-k}$ is the channel distorted version of $\varphi_{m n}$ and $\mathbf{n}=\left(n_{q}\right)_{q}$ is a discrete complex Additive White Gaussian Noise (AWGN), with zero mean and variance $N_{0}$.

The decision variable, denoted $\Lambda_{k l}$, on the transmitted symbol $a_{k l}$ is obtained by projecting $\mathbf{r}$ on the receive pulse $\boldsymbol{\psi}_{k l}$, such as:

$$
\Lambda_{k l}=\left\langle\boldsymbol{\psi}_{k l}, \mathbf{r}\right\rangle=\boldsymbol{\psi}_{k l}^{H} \mathbf{r},
$$

where $\boldsymbol{\psi}_{k l}=\left(\psi_{q-l N}\right)_{q} \odot\left(e^{j 2 \pi k q / Q}\right)_{q}$ is the time and frequency shifted version of the OFDM receive prototype waveform $\boldsymbol{\psi}=\left(\psi_{q}\right)_{q}$ and $\langle\cdot, \cdot\rangle$ is the Hermitian scalar product over the space of square-summable vectors.

\section{TR-POPS ALGORITHM}

The main objective of this part is to optimize the waveforms at the $\mathrm{Tx} / \mathrm{Rx}$ sides in our system based on TR technique. To this end, we adopt the POPS-OFDM principal [1]. This algorithm consists in maximizing the SINR for fixed synchronization imperfections and propagation channel.

Without loss of generality, we will focus on the SINR evaluation for the symbol $a_{00}$. Referring to (5), the decision variable on $a_{00}$ can be written as:

$\Lambda_{00}=a_{00}\left\langle\boldsymbol{\psi}_{00}, \tilde{\boldsymbol{\varphi}}_{00}\right\rangle+\sum_{(m, n) \neq(0,0)} a_{m n}\left\langle\boldsymbol{\psi}_{00}, \tilde{\boldsymbol{\varphi}}_{m n}\right\rangle+\left\langle\boldsymbol{\psi}_{00}, \mathbf{n}\right\rangle$

and it is composed of three terms. The first term is the useful part, the second term is the ISI and the last term presents the noise term. Their respective powers represent useful signal, interference and noise powers in the SINR and which we derive their closed form expressions in the sequel. This SINR will be the same for all other transmitted symbols.

\section{A. Average Useful, Interference and Noise Powers}

The useful term is denoted $U_{00}=a_{00}\left\langle\boldsymbol{\psi}_{00}, \tilde{\varphi}_{00}\right\rangle$. For a given realization of the channel, the average power of the useful term can be written as:

$$
P_{S}^{h}=\frac{E}{\|\boldsymbol{\varphi}\|^{2}}\left|\left\langle\boldsymbol{\psi}_{00}, \tilde{\boldsymbol{\varphi}}_{00}\right\rangle\right|^{2} .
$$

Thus, the useful power average over channel realizations is given by:

$$
P_{S}=\mathbb{E}\left[P_{S}^{h}\right]=\frac{E}{\|\boldsymbol{\varphi}\|^{2}} \mathbb{E}\left[\left|\left\langle\boldsymbol{\psi}_{00}, \tilde{\boldsymbol{\varphi}}_{00}\right\rangle\right|^{2}\right] .
$$

The interference term, $I_{00}=\sum_{(m, n) \neq(0,0)} a_{m n}\left\langle\boldsymbol{\psi}_{00}, \tilde{\boldsymbol{\varphi}}_{m n}\right\rangle$, results from the contribution of all other transmitted symbols $a_{m n}$, such as $(m, n) \neq(0,0)$.

For a given realization of the channel, the average power of the interference term can be written as:

$$
P_{I}^{h}=\frac{E}{\|\boldsymbol{\varphi}\|^{2}} \sum_{(m, n) \neq(0,0)}\left|\left\langle\boldsymbol{\psi}_{00}, \tilde{\boldsymbol{\varphi}}_{m n}\right\rangle\right|^{2} .
$$

Therefore, the interference power average over channel realizations has the following expression:

$$
P_{I}=\mathbb{E}\left[P_{I}^{h}\right]=\frac{E}{\|\varphi\|^{2}} \sum_{(m, n) \neq(0,0)} \mathbb{E}\left[\left|\left\langle\boldsymbol{\psi}_{00}, \tilde{\boldsymbol{\varphi}}_{m n}\right\rangle\right|^{2}\right],
$$

where

$$
\mathbb{E}\left[\left|\left\langle\boldsymbol{\psi}_{00}, \tilde{\boldsymbol{\varphi}}_{m n}\right\rangle\right|^{2}\right]=\boldsymbol{\psi}^{H} \mathbb{E}\left[\tilde{\boldsymbol{\varphi}}_{m n} \tilde{\boldsymbol{\varphi}}_{m n}^{H}\right] \boldsymbol{\psi}
$$

In the sequel, we consider a diffuse scattering function in the frequency domain, with a classical Doppler spectral density, decoupled from the dispersion in the time domain. So,

$$
\mathbb{E}\left[\left[\tilde{\boldsymbol{\varphi}}_{m n}\right]_{p}\left[\tilde{\boldsymbol{\varphi}}_{m n}^{H}\right]_{q}\right]=\sum_{k=-(K-1)}^{K-1}\left(\begin{array}{c}
\Pi_{k} J_{0}^{2}\left(\pi B_{d} T_{s}(p-q)\right) \\
\times\left[\boldsymbol{\varphi}_{m n}\right]_{p-k}\left[\boldsymbol{\varphi}_{m n}\right]_{q-k}^{*}
\end{array}\right),
$$

where $B_{d}$ is the Doppler Spread, $J_{0}(\cdot)$ is the Bessel function of the first kind of order zero and

$$
\Pi_{k}= \begin{cases}\sum_{l=0}^{K-1} \pi_{l}^{2}+\left(\sum_{l=0}^{K-1} \pi_{l}\right)^{2}, & \text { if } k=0 \\ \sum_{l=0}^{K-1-|k|} \pi_{l} \pi_{l+|k|} & , \text { else }\end{cases}
$$

is the average power of the global channel. Then the average useful and interference powers have the following expressions:

$$
P_{S}=\frac{E}{\|\boldsymbol{\varphi}\|^{2}} \boldsymbol{\psi}^{H} \mathbf{K S}^{\boldsymbol{\varphi}} \boldsymbol{\psi} \quad \text { and } \quad P_{I}=\frac{E}{\|\boldsymbol{\varphi}\|^{2}} \boldsymbol{\psi}^{H} \mathbf{K} \mathbf{I}^{\boldsymbol{\varphi}} \boldsymbol{\psi}
$$

where $\mathbf{K S}^{\varphi}$ and $\mathbf{K I}^{\varphi}$ are Hermitian, symmetric, positive and semidefinite matrices:

$$
\mathbf{K S}^{\boldsymbol{\varphi}}=\left(\sum_{k=-(K-1)}^{K-1} \Pi_{k} \boldsymbol{\Sigma}_{k}\left(\varphi \varphi^{H}\right)\right) \odot \boldsymbol{\Lambda}
$$

and

$\mathbf{K I}^{\boldsymbol{\varphi}}=\left[\left(\sum_{n} \boldsymbol{\Sigma}_{n N}\left(\sum_{k=-(K-1)}^{K-1} \Pi_{k} \boldsymbol{\Sigma}_{k}\left(\varphi \varphi^{H}\right)\right)\right) \odot \boldsymbol{\Omega}\right]-\mathbf{K S}^{\varphi}$.

The entries of matrices $\boldsymbol{\Lambda}$ and $\boldsymbol{\Omega}$ are defined as:

$$
\Lambda_{p q}=J_{0}^{2}\left(\pi B_{d} T_{s}(p-q)\right)
$$

and

$$
\Omega_{p q}=\left\{\begin{array}{lc}
Q J_{0}^{2}\left(\pi B_{d} T_{s}(p-q)\right) & \text { if }(p-q) \bmod Q=0, \\
0, & \text { else },
\end{array}\right.
$$

with $p, q \in \mathbb{Z}$.

The noise term is given by $N_{00}=\left\langle\boldsymbol{\psi}_{00}, \boldsymbol{n}\right\rangle$. Thus, the noise power average is the following:

$$
P_{N}=\mathbb{E}\left[\left|\left\langle\boldsymbol{\psi}_{00}, \mathbf{n}\right\rangle\right|^{2}\right]=\boldsymbol{\psi}^{H} \mathbb{E}\left[\mathbf{n} \mathbf{n}^{H}\right] \boldsymbol{\psi} .
$$

As the noise is supposed to be white, its covariance matrix is equal to $\mathbf{R}_{\mathbf{n n}}=\mathbb{E}\left[\mathbf{n n}^{H}\right]=N_{0} \mathbf{I}$, where $\mathbf{I}$ is the identity matrix. Consequently,

$$
P_{N}=N_{0}\|\boldsymbol{\psi}\|^{2} .
$$




\section{B. Optimization Technique}

The SINR expression is the following:

$$
S I N R=\frac{P_{S}}{P_{I}+P_{N}}=\frac{\psi^{H} \mathbf{K S}^{\varphi} \psi}{\psi^{H} \mathbf{K I N}^{\varphi} \psi},
$$

where $\mathbf{K I N}^{\varphi}=\mathbf{K I}^{\varphi}+\frac{N_{0}}{E}\|\varphi\|^{2} \mathbf{I}$.

Our optimization technique is an iterative algorithm where we maximize alternately the $\mathrm{Rx}$ waveform $\psi$, for a given $\mathrm{Tx}$ waveform $\varphi$, and the Tx waveform $\varphi$, for a given $\operatorname{Rx}$ waveform $\psi$.

Note that (13) can also be written as:

$$
S I N R=\frac{\varphi^{H} \mathbf{K S}^{\psi} \varphi}{\varphi^{H} \mathbf{K I N}^{\psi} \varphi},
$$

where $\mathbf{K S}^{\psi}$ and $\mathbf{K I N}^{\psi}$ are expressed as:

$$
\mathbf{K S}^{\boldsymbol{\psi}}=\left(\sum_{k=-(K-1)}^{K-1} \Pi_{k} \boldsymbol{\Sigma}_{k}\left(\boldsymbol{\psi} \boldsymbol{\psi}^{H}\right)\right) \odot \boldsymbol{\Lambda}
$$

and

$$
\mathbf{K I N}^{\boldsymbol{\psi}}=\mathbf{K I}^{\boldsymbol{\psi}}+\frac{N_{0}}{E}\|\boldsymbol{\psi}\|^{2} \mathbf{I}
$$

with

$\mathbf{K I}^{\boldsymbol{\psi}}=\left(\sum_{n} \boldsymbol{\Sigma}_{n N}\left(\sum_{k=-(K-1)}^{K-1} \Pi_{k} \boldsymbol{\Sigma}_{k}\left(\boldsymbol{\psi} \boldsymbol{\psi}^{H}\right)\right)\right) \boldsymbol{\Omega}-\mathbf{K S}^{\boldsymbol{\psi}}$.

Thus, the optimization problem is equivalent to maximizing a generalized Rayleigh quotient. As $\frac{N_{0}}{E}\|\varphi\|^{2}>0$, we can affirm that $\mathrm{KIN}^{\varphi}$ is always invertible and relatively wellconditioned.

The main steps of the proposed algorithm, presented by Figure 1 , are the following:

- Step 1: We initialize the algorithm with $\varphi^{(0)}$,

- Step 2: For the iteration $(i)$, we compute $\psi^{(i)}$ as the eigenvector of $\left(\mathbf{K I N}^{\varphi^{(i)}}\right)^{-1} \mathbf{K S}^{\varphi^{(i)}}$ with maximum eigenvalue,

- Step 3: For the obtained $\psi^{(i)}$, we determine $\varphi^{(i+1)}$ as the eigenvector of $\left(\mathbf{K I N}^{\psi^{(i)}}\right)^{-1} \mathbf{K} \mathbf{S}^{\psi^{(i)}}$ with maximum eigenvalue,

- Step 4: We proceed to the next iteration, $(i+1)$,

- Step 5: We stop the iterations when we obtain a negligible variation of SINR.

We note that eig, used in Figure 1, is a function that returns the eigenvector of a square matrix with the largest eigenvalue.

\section{Simulation Results}

In this section, the performances of the proposed TR-POPS technique are evaluated. To show the gain in terms of SINR and Power Spectral Density (PSD), a comparison with POPSOFDM and conventional OFDM with TR is also realized.

The results of POPS-OFDM algorithm applied to our system based on TR technique are carried out for a discrete timefrequency lattice. The optimal Tx/Rx waveform couple maximizing the SINR, $\left(\varphi_{\text {opt }}, \boldsymbol{\psi}_{\text {opt }}\right)$, is evaluated for a Gaussian

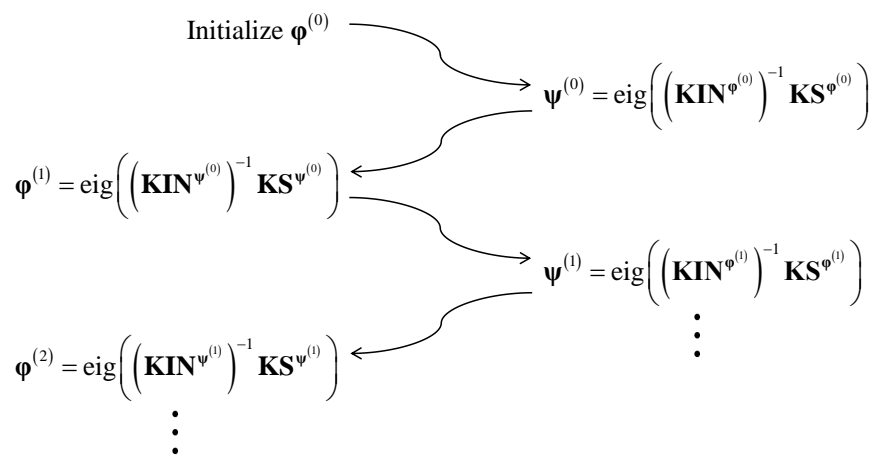

Figure 1: Optimization philosophy.

initialization waveform $\varphi^{(0)}$.We presume having an exponential truncated decaying model.

Figure 2 presents the evolution of the SINR versus the normalized Doppler spread $B_{d} / F$ for a normalized channel delay spread $T_{m} / T$ where $Q=128, N=144$, a lattice density is equal to $8 / 9$ and for a waveform support duration $D=3 T$. The obtained results demonstrate that TR-POPSOFDM approach improves the SINR with a gain of $2.3 \mathrm{~dB}$ for $B_{d} / F=0.1$ compared with POPS-OFDM and a gain that can reach $5.2 \mathrm{~dB}$ for $B_{d} / F=0.02$ compared with conventional OFDM with TR. Moreover, this figure is a mean to find the adequate couple $(T, F)$ of an envisaged application to insure the desired transmission quality.

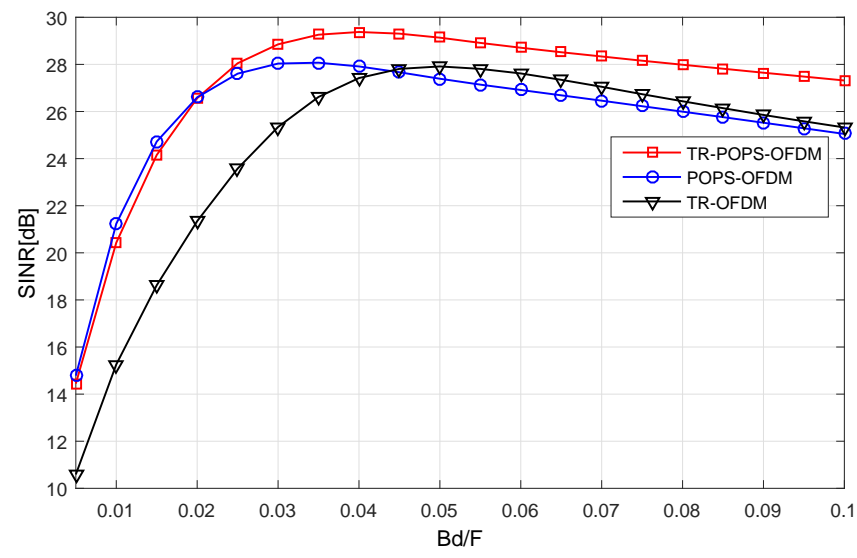

Figure 2: Optimized SINR as a function of $\frac{B_{d}}{F}$ for $Q=128$, $S N R=30 \mathrm{~dB}, B_{d} T_{m}=10^{-3}$ and $D=3 T$.

Figure 3 illustrates the effect of TR by showing the evolution of the SINR with respect to the time-frequency parameter $F T$. As in Figure 2, our proposed system outperforms the POPS-OFDM system and conventional OFDM with TR. The presented results reveal an increase in the obtained SINR that can reach $1.45 \mathrm{~dB}$ for $F T=1+\frac{8}{128}$ compared with POPSOFDM and an increase of $4.5 \mathrm{~dB}$ for $F T=1+\frac{48}{128}$ compared to conventional OFDM with TR.

Figure 4 shows that, thanks to the TR technique, the obtained optimal transmit waveform, $\varphi_{\text {opt }}$, reduces the OOB 


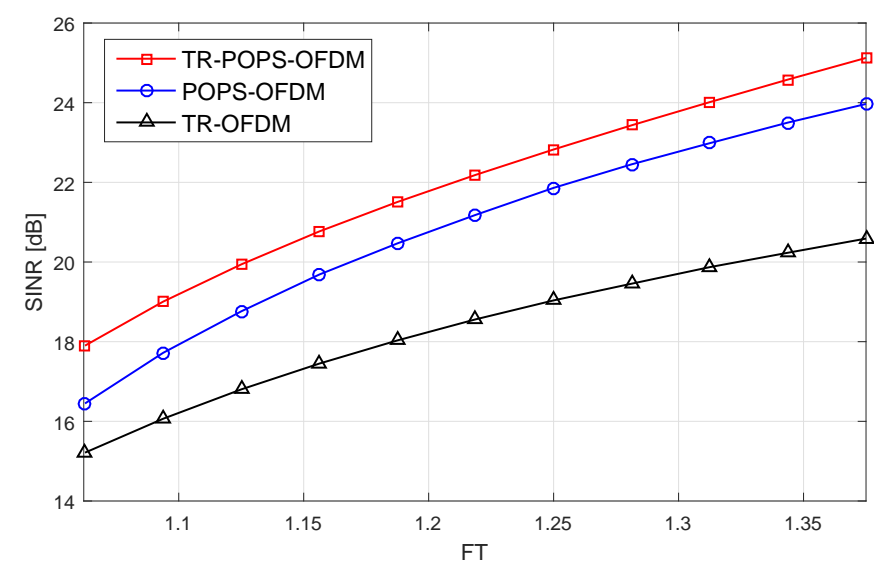

Figure 3: SINR versus $F T$ for $Q=128, S N R=30 \mathrm{~dB}$, $B_{d} T_{m}=10^{-2}$ and $D=3 T$.

emissions by about $40 \mathrm{~dB}$ compared to the POPS-OFDM system without TR.

We present in Figure 5 the Tx/Rx waveforms, $\varphi_{o p t}$ and $\boldsymbol{\psi}_{\text {opt }}$, corresponding to the optimal SINR for $Q=128, N=$ $144, F T=1+\frac{16}{128}, B_{d} T_{m}=0.001$ and $D=3 T$. Since the channel is characterized by an Hermitian and symmetric response thanks to the TR effect, we obtain identical Tx/Rx waveforms as it is illustrated in this figure.

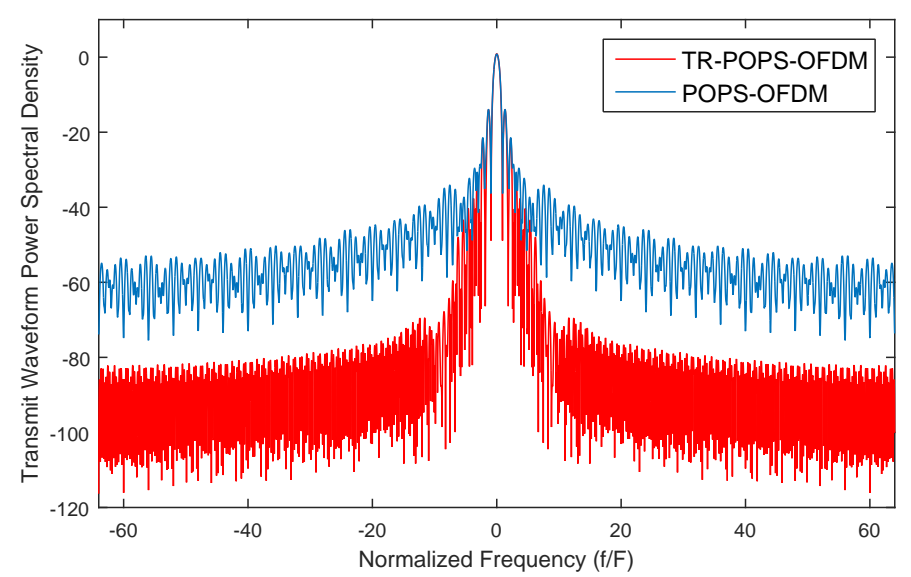

Figure 4: PSD of the optimized transmit waveform for $Q=$ $128, S N R=30 \mathrm{~dB}, B_{d} T_{m}=10^{-3}, F T=1.25$ and $D=3 T$.

\section{CONCLUSION}

In this paper, we studied the association of POPS-OFDM algorithm with TR precoding technique to design novel waveforms for $5 \mathrm{G}$ systems. To this end, we presented the corresponding system model and we derived the analytical SINR expression. Despite the additional complexity of applying the combination process, simulation results showed that the proposed approach offers a highly flexible behavior and better performances in terms of maximization of the SINR and reduction of the ISI/ICI. Another possible challenging research

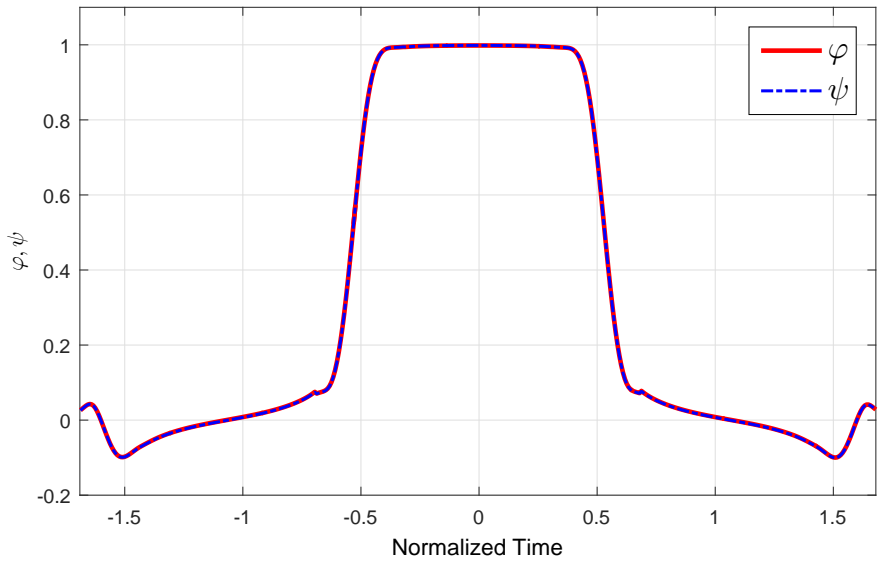

Figure 5: Tx/Rx optimized waveforms for $D=3 T$.

axis consists in applying this combination in MIMO-OFDM and FBMC/OQAM systems.

\section{REFERENCES}

[1] M. Siala, F. Abdelkefi, and Z. Hraiech, "Novel Algorithms for Optimal Waveforms Design in Multicarrier Systems," IEEE Wireless Communications and Networking Conference (WCNC 2014), Istanbul, Turkey, Apr. 2014.

[2] T. Yunzheng, L. Long, L. Shang, and Z. Zhi, "A Survey: Several Technologies of Non-Orthogonal Transmission for 5G," China Соттиnications, Oct. 2015.

[3] F.-L. Luo and C. J. Zhang, Signal Processing for 5G: Algorithms and Implementations, John Wiley \& Sons, United Kingdom, Oct. 2016.

[4] F. Schaich and T. Wild, "Waveform Contenders for 5G - OFDM vs. FBMC vs. UFMC," Proceedings of 6th International Symposium on Communications, Control, and Signal Processing (ISCCSP), pp. 457-460, May 2014.

[5] G. Wunder, P. Jung, M. Kasparick, T. Wild, F. Schaich, Y. Chen, S. ten Brink, I. Gaspar, N. Michailow, A. Festag, L. Mendes, N. Cassiau, D. Ktnas, M. Dryjanski, S. Pietrzyk, P. Vago, and F. Wiedmann, "5GNOW: Non-Orthogonal, Asynchronous Waveforms for Future Mobile Applications," IEEE Communications Magazine, Feb. 2014.

[6] Z. Hraiech, F. Abdelkefi, and M. Siala, "POPS-OFDM: Ping-pong Optimized Pulse Shaping-OFDM for 5G Systems," IEEE International Conference on Communications (ICC), London, June 2015.

[7] T. Dubois, M. Hélard, M. Crussière, and C. Germond, "Performance of Time Reversal Precoding Technique for MISO-OFDM Systems," EURASIP Journal on Wireless Communications and Networking, Nov. 2013.

[8] M. Fink, "Time Reversal of Ultrasonic Fields - Part I: Basic Principles," IEEEtran on Ultrasonics, Ferroelectrics and Frequency Control, Sep. 1992.

[9] G. Lerosey, J. de Rosny, A. Tourin, A. Derode, G. Montaldo, and M. Fink, "Time Reversal of Electromagnetic Waves," Phys. Rev. Lett., May 2004. 